\title{
Versión Abreviada del Inventario de Perspectiva Temporal de Zimbardo para Buenos Aires
}

\author{
Short Version of the Zimbardo Time Perspective Inventory for Buenos Aires
}

\author{
Guadalupe Germano ${ }^{1}$ y María Elena BRenlla ${ }^{2}$
}

\begin{abstract}
Resumen
Se presenta una versión breve del Inventario de Perspectiva Temporal de Zimbardo (ZTPI) para Buenos Aires que fue construida a partir de la adaptación argentina de 56 ítems y que fue respondida por 343 personas junto con la escala de Malestar Psicológico y la de Autocontrol. A partir de un análisis paralelo clásico y un análisis factorial exploratorio se verificó la estructura original de cinco factores y se llegó a una versión del ZTPI breve (ZTPI-B) de 29 ítems. En un segundo estudio 272 personas completaron el ZTPI-B y las mismas medidas externas. El análisis factorial semiconfirmatorio mostró un muy buen ajuste del modelo mientras que los análisis de validez externa mostraron asociaciones significativas entre pasado negativo, presente fatalista y malestar psicológico y futuro y autocontrol. Los resultados indican buenas evidencias de consistencia interna y de validez de criterio, de contenido y de constructo.
\end{abstract}

Palabras clave: perspectiva temporal, ZTPI, malestar psicológico, autocontrol

\begin{abstract}
A brief version of the Zimbardo Temporary Perspective Inventory (ZTPI) for Buenos Aires is presented. It was constructed from the Argentine adaptation of 56 items which was answered by 343 people along with the scale of Psychological Distress and Self-control. From a classic parallel analysis and an exploratory factor analysis, the original five-factor factor structure was verified and a short version of the ZTPI (ZTPI-B) of 29 items was achieved. In a second study 272 people completed the ZTPI-B and the same external measures. The semiconfirmatory factor analysis showed a very good adjustment of the model while external validity analyzes showed significant associations between negative past, fatalistic present and psychological distress and future and self-control. The results indicate satisfactory evidence of internal consistency and validity of criteria and content.
\end{abstract}

Keywords: time perspective, ZTPI, psychological distress, self-control

\footnotetext{
${ }^{1}$ Lic. en Psicología. Becaria doctoral CONICET en Centro de Investigaciones en Psicología y Psicopedagogía. Pontificia Universidad Católica Argentina. Av. Alicia Moreau de Justo 1600 (C1107AAZ), CABA, Argentina. Tel.: +5401143490200. Correo: guadalupe_germano@uca.edu.ar

${ }^{2}$ Dra. en Psicología. Investigadora en Centro de Investigaciones en Psicología y Psicopedagogía. Pontificia Universidad Católica Argentina. 


\section{Introducción}

En 1999 Zimbardo y Boyd comienzan un estudio exhaustivo de la Perspectiva Temporal cuyo enfoque general abreva en la teoría propuesta por Lewin (1942) - que redundó en una nueva definición al concepto y en el desarrollo de un instrumento para su medición. Para los autores, la Perspectiva Temporal se entiende como "el proceso no consciente, mediante el cual el flujo de las experiencias personales y sociales se encuadran en categorías temporales que ayudan a dar orden, coherencia y significado a esas experiencias" (p. 1271) y representa una dimensión psicológica básica del tiempo que refiere a la forma en que cada individuo se relaciona con él. Alude al proceso a través del cual las distintas experiencias sociales e individuales son codificadas en diferentes marcos temporales -pasado negativo, pasado positivo, presente hedonista, presente fatalista y futuro- y cómo estos marcos ayudan a ordenar la experiencia y darle coherencia y sentido a nuestras vidas. De esta manera, la perspectiva temporal es utilizada al codificar y recordar eventos así como en la formación de objetivos, expectativas y escenarios imaginarios (Zimbardo \& Boyd, 1999).

Los autores desarrollaron un cuestionario para evaluar de manera cuantificable la Perspectiva Temporal: el Inventario de Perspectiva Temporal de Zimbardo (ZTPI, por sus iniciales en inglés). El ZTPI es un cuestionario auto-administrable que consta de 56 ítems con un formato de respuesta de tipo Likert con cinco opciones. A través de sendas medidas, se evalúan los cinco factores que componen la perspectiva temporal. El perfil resultante permite saber si la persona tiene una perspectiva temporal balanceada o si prevalece una orientación por sobre las demás. La perspectiva temporal balanceada es la que le permite al sujeto cambiar flexiblemente su marco temporal. La necesidad de cambiar se da, entre otros aspectos, por las demandas de la situación y las propias preferencias. En cambio, cuando el sujeto sobre-enfatiza una dimensión particular se produce un sesgo que podría implicar utilizar ese marco temporal excesivamente hasta poder transformarse en su estilo de funcionamiento, dejando de lado los otros marcos temporales (Zimbardo \& Boyd, 1999).

El cuestionario de 56 ítems fue validado en una muestra de estudiantes estadounidenses $(N=606) . \quad$ Se realizó un análisis factorial exploratorio con un $\mathrm{KMO}=.83$ y se aplicó el método de rotación Varimax que arrojó cinco factores que explicaron el $36 \%$ de la varianza. Los cinco factores encontrados fueron: Pasado Negativo, Presente Hedonista, Futuro, Pasado Positivo y Presente Fatalista. Para todos los factores se hallaron buenos índices de consistencia interna, ya que los valores de alfa de Cronbach van desde .74 hasta .82 (Zimbardo \& Boyd, 1999).

Los resultados de investigaciones confirman que los distintos tipos de perspectiva temporal están significativamente asociados a diferentes aspectos psicológicos. Entre ellos, el pasado positivo se asocia con alta autoestima, energía y felicidad; el pasado negativo con baja autoestima, agresión y depresión (Stolarski, Matthews, Postek, Zimbardo, \& Bitner, 2014); el presente hedonista con conductas de riesgo y adicciones y búsqueda de novedad (Klingeman, 2001); el presente fatalista con depresión, ansiedad y agresión (Zimbardo \& Boyd, 1999, 2008) y el futuro con la estabilidad emocional, y la capacidad de ser diligente y establecer metas (Zhang \& Howell, 2011). Además, se ha demostrado que el perfil de perspectiva temporal balanceada correlaciona positivamente con la salud, mientras que la prevalencia de un factor, como por ejemplo el pasado negativo, lo hace de manera negativa (Oyanadel \& Buela-Casal, 2010).

Sircova y cols. (2014) realizaron un estudio en donde evaluaron la equivalencia estructural del ZTPI en 26 muestras de 24 países. Se probó que el cuestionario es válido y fiable para evaluar las diferencias individuales en perspectiva temporal a través de las cinco categorías propuestas por los autores originales. Esto demuestra que el ZTPI fue y es muy utilizado en la investigación psicológica y que es considerado un gold standard para su propósito de evaluación. Cabe destacar que los estudios que informan la adaptación y validación del ZTPI siguen, en su mayoría, el análisis de datos propuesto por los autores originales.

Respecto de la adaptación al idioma español, 
Tabla 1. Revisión de las distintas versiones del ZTPI en español

\begin{tabular}{|c|c|c|c|c|c|c|}
\hline Autores & País & $\begin{array}{c}\text { Edad de los } \\
\text { participantes }\end{array}$ & Participantes & $\alpha$ de Cronbach & $\begin{array}{r}\text { Varianza } \\
\text { explicada }\end{array}$ & Análisis \\
\hline $\begin{array}{l}\text { Díaz-Morales } \\
\text { (2006) }\end{array}$ & España & 19 a 67 & 756 & $\begin{array}{l}\text { Pasado Negativo }=.80 \\
\text { Presente } \\
\text { Hedonista }=.79 \\
\text { Futuro }=.70 \\
\text { Pasado Positivo }=.70 \\
\text { Presente Fatalista }=.64\end{array}$ & 33.82 & $\begin{array}{c}\text { ACP } \\
\text { (Varimax) }\end{array}$ \\
\hline $\begin{array}{l}\text { Corral-Verdugo. } \\
\text { Fraijo-Sing y } \\
\text { Pinheiro (2006) }\end{array}$ & México & 12 a 69 & 300 & $\begin{array}{l}\text { Pasado }=.77 \\
\text { Presente }=.80 \\
\text { Futuro }=.75\end{array}$ & - & $\mathrm{AFC}$ \\
\hline $\begin{array}{l}\text { Oyandel. Buela- } \\
\text { Casal y Pérez- } \\
\text { Fortis (2014) }\end{array}$ & Chile & 18 a 70 & 604 & $\begin{array}{l}\text { Pasado Negativo }=.80 \\
\text { Presente } \\
\text { Hedonista }=.79 \\
\text { Futuro }=.80 \\
\text { Pasado Positivo }=.59 \\
\text { Presente Fatalista }=.74 \\
\text { Pasado Negativo }=80\end{array}$ & 33 & $\begin{array}{c}\mathrm{ACP} \\
\text { (Varimax) }\end{array}$ \\
\hline $\begin{array}{l}\text { Galarraga y } \\
\text { Stover (2016) }\end{array}$ & Argentina & 15 a 20 & 320 & $\begin{array}{l}\text { Presente } \\
\text { Hedonista }=.78 \\
\text { Futuro }=.68 \\
\text { Presente Fatalista }=.63\end{array}$ & 37.94 & $\begin{array}{c}\text { ACP } \\
(\text { Varimax) }\end{array}$ \\
\hline $\begin{array}{l}\text { González- } \\
\text { Lomelí, } \\
\text { Maytorena- } \\
\text { Noriega, } \\
\text { Cárdenas-Niño } \\
\text { y Tapia- } \\
\text { Fonllem (2018) }\end{array}$ & Colombia & universitarios & 770 & $\begin{array}{l}\text { Pasado Negativo }=.71 \\
\text { Presente } \\
\text { Hedonista }=.65 \\
\text { Futuro }=.63 \\
\text { Pasado Positivo }=.60 \\
\text { Presente Fatalista }=.61\end{array}$ & - & $\mathrm{AFC}$ \\
\hline $\begin{array}{l}\text { Brenlla. } \\
\text { Germano y } \\
\text { Zapater (2019) }\end{array}$ & Argentina & 19 a 65 & 340 & $\begin{array}{l}\text { Pasado Negativo }=.78 \\
\text { Presente } \\
\text { Hedonista }=.78 \\
\text { Futuro }=.65 \\
\text { Pasado Positivo }=.59 \\
\text { Presente Fatalista }=.66\end{array}$ & 36 & $\begin{array}{c}\mathrm{ACP} \\
\text { (Varimax) }\end{array}$ \\
\hline
\end{tabular}

el primero en realizarla fue Díaz-Morales (2006) quien llevó a cabo un estudio en población española que mostró una estructura factorial de cinco factores que se asemeja a la obtenida en la muestra estadounidense. Sin embargo, se hallaron algunas diferencias en los factores pasado positivo y presente fatalista que podrían atribuirse a diferencias culturales. A partir de la versión de Díaz Morales (2006) se realizaron estudios en diversos países latinoamericanos. Este proceso es necesario debido a que la constatación de equivalencias lingüísticas y culturales de los test deben ser tenidas en cuenta a la hora de evaluar propiedades psicométricas de una prueba (Martínez-Arias, 1995).

Actualmente el ZTPI se encuentra adaptado en México (Corral-Verdugo, Fraijo-Sing, \& Pinheiro, 2006), Chile (Oyanadel, Buela-Casal, \& PérezFortis, 2014), Argentina (Galarraga \& Stover, 2016; Brenlla, Germano, \& Zapater, 2019) y Colombia (González-Lomelí, Maytorena-Noriega, Cárdenas-Niño, \& Tapia-Fonllem, 2018). En la
Tabla 1 se pueden ver los principales resultados informados en cada adaptación.

Como puede observarse, en Argentina se realizaron dos estudios para analizar las propiedades psicométricas del ZTPI. Uno referido a adolescentes (Galarraga \& Stover, 2016) y otro a adultos (Brenlla et al, 2019). Respecto del primero, la muestra estuvo compuesta por estudiantes de nivel medio de Buenos Aires lo cual difiere del rango etario considerado en la versión original. Además, en esta adaptación se aisló una estructura temporal de cuatro dimensiones en lugar de las cinco propuestas originariamente, lo que dio lugar a una reducción de la cantidad de ítems. Las autoras explicaron que estas diferencias se deben fundamentalmente a cambios culturales y a la diferencia de la edad de los evaluados. Por otro lado, se realizó la adaptación del ZTPI con adultos (Brenlla et al., 2019). Este estudio mostró resultados que corroboran la estructura de cinco componentes del 
estudio original así como también evidencias satisfactorias de validez externa y de fiabilidad.

En el actual estudio se propone una versión breve del Inventario de Perspectiva Temporal de Zimbardo (ZTPI-B) para la población de Buenos Aires. Ahora bien, ¿por qué es necesario acortar la prueba? En primer lugar, y como se mencionó previamente, la escala original consta de 56 ítems lo que implica que completarla lleva un tiempo considerable. Cuando se utiliza una batería de cuestionarios para investigación o clínica se recomienda usar inventarios cortos ya que resultan menos cansadores para los participantes y porque al completar pruebas largas, las personas lo hacen de manera menos fiable. Por lo tanto, las pruebas deben ser lo más breves posibles (Worthington \& Whittaker, 2006). En este sentido, se han realizado diversas versiones abreviadas del ZTPI para distintas poblaciones, pero ninguna en español.

Las numerosas versiones cortas del cuestionario demuestran que el ZTPI es muy utilizado. Al considerar los resultados se pueden observar diferencias. Entre ellas se encuentran, en primer lugar, la cantidad total de ítems del cuestionario van de 15 a 36 . En segundo lugar, en varios estudios la escala presente fatalista mostró baja fiabilidad por lo que algunos autores decidieron eliminarla. Por último, también varía la cantidad de ítems para cada factor (para una revisión exhaustiva de estas diferencias véase Przepiorka, Sobol-Kwapinska, \& Jankowski, 2016).

El objetivo del estudio fue desarrollar y validar una versión breve del ZTPI para población de Buenos Aires. Para ello, en primer lugar y de acuerdo con los criterios sugeridos por Marsh, Martin y Jackson (2010) para realizar versiones abreviadas, se confeccionó a una versión corta preliminar del inventario utilizando los datos informados en la adaptación para Buenos Aires del ZTPI. Se realizó un primer estudio en el cual esta versión de 41 ítems fue administrada a los participantes y a partir de ello se realizaron los análisis pertinentes para llegar a la versión abreviada final que fue completada por otros participantes en un segundo estudio para analizar su estructura factorial.

\section{Método}

Esta investigación fue de tipo cuantitativa, descriptiva y correlacional con un diseño de tipo instrumental, según la clasificación de Montero y León (2007). Para la abreviación del instrumento a la realidad cultural de Buenos Aires se utilizaron los criterios desarrollados por Marsh, Martin y Jackson (2010).

\section{Estudio 1}

\section{Participantes}

La muestra estuvo compuesta por 343 personas, $65 \%$ mujeres, de nacionalidad argentina. Las edades estuvieron comprendidas entre 18 y 65 años $\quad(M E=27.5 ; \quad D E=11.8) . \quad$ Todos los participantes viven en zonas urbanas de Buenos Aires, $52 \%$ en Gran Buenos Aires y $48 \%$ en la Ciudad Autónoma de Buenos Aires. En cuanto al nivel de estudios alcanzados $36 \%$ tiene terciario/universitario incompleto, 29\% secundario completo, $27 \%$ terciario/universitario completo, $5 \%$ posgrado/doctorado y $2 \%$ secundario incompleto. Participaron del estudio entre marzo y noviembre de 2018.

\section{Instrumentos}

Datos sociodemográficos. Cuestionario construido ad hoc en el que se indagó la edad, el sexo, la nacionalidad, el lugar de residencia y el nivel máximo de educación alcanzado.

Inventario de Perspectiva Temporal de Zimbardo (ZTPI). Evalúa la Perspectiva Temporal en cinco dominios. Fue desarrollada por Zimbardo y Boyd (1999) y consta de 56 ítems con cinco opciones de respuesta en escala tipo Likert. Para este estudio se utilizó la versión de 41 ítems realizada a partir del análisis informado en la adaptación del ZTPI para Buenos Aires (Brenlla et al., 2019). Los ítems evalúan los cinco factores propuestos por los autores originales: pasado negativo (9 ítems), presente hedonista (10 ítems), futuro (7 ítems), pasado positivo ( 9 ítems) y presente fatalista (7 ítems).

Escala de Malestar Psicológico de Kessler K10. Desarrollada por Kessler y Mrozeck (1994), consta de 10 ítems con formato de respuesta tipo Likert de cinco puntos. Evalúa el riesgo de 
presentar malestar psicológico inespecífico, síntomas de ansiedad o depresión, en el último mes. Se utilizó la adaptación argentina desarrollada por Brenlla y Aranguren (2010), en la cual la confiabilidad evaluada mediante el coeficiente alfa de Cronbach fue igual a .80 .

Escala de Autocontrol (EAC). Es uno de los instrumentos más utilizados para evaluar autoncotrol. Fue desarrollada por Tangney, Baumeister y Boone (2004). Está compuesta por 36 ítems, con cinco opciones de respuesta de formato Likert. Evalúa el grado de autocontrol que presentan las personas. Se utilizó la adaptación argentina desarrollada por Garrido, Cupani y Arbach (2017) cuya confiabilidad evaluada mediante el coeficiente alfa de Cronbach fue igual a .85 .

\section{Procedimiento}

Las personas fueron evaluadas individualmente $\mathrm{o}$ en pequeños grupos. Los participantes aceptaron el consentimiento informado correspondiente donde se les garantizó la confidencialidad de los datos que se obtuvieran. Luego se aplicaron los instrumentos en el siguiente orden: cuestionario de datos sociodemográficos, ZTPI, K-10, EAC. Todos los cuestionarios fueron auto-administrados. No existió compensación económica o de otro tipo para los participantes.

\section{Análisis de datos}

Se calcularon las medidas descriptivas de cada factor utilizando el paquete estadístico IBM SPSS 25. También se calculó la consistencia interna de cada factor con el estadístico alfa de Cronbach y se hicieron los análisis de correlación con medidas externas calculando el estadístico $r$ de Pearson. Luego, utilizando el programa estadístico FACTOR 9.2 (Lorenzo-Seva \& Ferrando, 2013) se realizó un análisis paralelo clásico basado en el análisis de componentes principales (Horn, 1965) y el análisis factorial exploratorio en la muestra total a partir del método de extracción Unweighted Least Squares (ULS).

\section{Resultados}

En primer lugar, se revisó el estudio de la versión original del ZTPI en el cual se notó que 15 de los 56 ítems originales presentaron cargas factoriales bajas, es decir menores a .30; cargas compartidas, esto es, saturaciones superiores a .30 en dos o más factores; o contradictorias, cargas significativas en ítems que evaluaban el rasgo contradictorio al que se quería evaluar. Se recomendó tener en cuenta esta evidencia en futuros estudios (Brenlla et al., 2019).

A continuación, se indican los resultados más salientes ya que son de relevancia para justificar la reducción del inventario. Se eliminó el ítem nueve, 'No me preocupa si las cosas no se hacen a tiempo', perteneciente al factor futuro por tener baja carga factorial, lo cual se deba probablemente a la redacción con doble negación. Por otro lado, se eliminaron cinco ítems del presente hedonista y uno de futuro por presentar cargas compartidas; dos del presente fatalista, uno del futuro, uno del presente hedonista y uno del pasado negativo por cargar en un factor contradictorio; y se eliminaron dos ítems del futuro y uno del pasado positivo por tener solamente cargas negativas y compartidas. Esto permitió obtener una versión preliminar más breve del ZTPI que constó de 41 ítems basada en la supresión de los ítems con un comportamiento psicométrico anómalo en el análisis de la versión original. A partir de esto se realizó el trabajo de campo $(N=343)$ para obtener la versión abreviada final del ZTPI-B.

Para evaluar la confiabilidad de la prueba se calculó el coeficiente alfa de Cronbach para cada una de las escalas. Los valores obtenidos fueron entre .63 y .80 (ver Tabla 2). Por otro lado, se realizó un análisis de validez externa con el fin de evaluar si la escala compuesta por 41 ítems continuaba evaluando lo mismo que la escala original. El factor pasado negativo correlacionó negativamente con la escala de autocontrol ( $r=-.39$; $p<.05)$ y positivamente con la escala de malestar psicológico $(r=.49 ; \quad p<.05)$. el factor presente fatalista correlacionó negativamente con la escala de autocontrol $(r=-.26 ; p<.05)$ y positivamente con la escala de malestar psicológico $(r=.50 ; p<.05)$. El factor pasado positivo correlacionó negativamente con la escala de malestar psicológico ( $r=-.18$; $p<.05)$. El factor futuro correlacionó positivamente con la escala de autocontrol $(r=.48 ; p<.05)$. Por último, el factor presente hedonista correlacionó de manera negativa con la escala de autocontrol $(r=$ $-.45 ; p<.05)$. Se calcularon las medidas descriptivas de cada ítem. Los valores de asimetría y curtosis 
Tabla 2. Cargas factoriales del ZTPI de 41 ítems - Modelo 1

\begin{tabular}{|c|c|c|c|c|c|c|c|c|}
\hline \multirow[t]{2}{*}{ Factor e ítem } & \multicolumn{8}{|c|}{ Factores } \\
\hline & $\begin{array}{c}\text { Pasado } \\
\text { Negativo }\end{array}$ & $\begin{array}{l}\text { Presente } \\
\text { Hedonista }\end{array}$ & Futuro & $\begin{array}{l}\text { Pasado } \\
\text { Positivo }\end{array}$ & $\begin{array}{l}\text { Presente } \\
\text { Fatalista }\end{array}$ & $\begin{array}{c}\text { Factor } \\
6\end{array}$ & $\begin{array}{l}\text { Alfa si se } \\
\text { elimina el } \\
\text { elemento }\end{array}$ & $\begin{array}{l}\text { Correlación } \\
\text { ítem-factor }\end{array}$ \\
\hline \multicolumn{9}{|l|}{ Pasado Negativo } \\
\hline Ítem 3 & .49 & -.08 & -.05 & -.09 & .01 & .09 & .77 & .60 \\
\hline Ítem 12 & .53 & .02 & -.01 & -.10 & .04 & -.04 & .77 & .63 \\
\hline Ítem 17 & .28 & .23 & .13 & -.41 & -.40 & -.07 & .78 & .60 \\
\hline Ítem 21 & .46 & .17 & -.01 & -.05 & .06 & .04 & .77 & .65 \\
\hline Ítem 25. & .23 & .01 & .03 & -.11 & .25 & .01 & .81 & .29 \\
\hline Ítem 26 & .38 & .08 & .09 & -.38 & .02 & .07 & .77 & .66 \\
\hline Ítem 28 & .72 & .11 & -.14 & .19 & -.08 & .02 & .77 & .65 \\
\hline Ítem 3838 & .67 & .04 & .07 & -.23 & -.16 & -.13 & .76 & .71 \\
\hline Ítem 40 & .53 & -.13 & -.06 & -.02 & -.01 & .31 & .77 & .62 \\
\hline \multicolumn{9}{|l|}{ Presente Hedonista } \\
\hline Ítem 1 & .21 & .28 & .03 & .21 & -.05 & .23 & .69 & .45 \\
\hline Ítem 5 & .13 & .48 & -.13 & .15 & .11 & -.08 & .66 & .58 \\
\hline Ítem 8 & .02 & .21 & .20 & .07 & .15 & -.04 & .70 & .51 \\
\hline Ítem 14 & .01 & .36 & .18 & .21 & -.09 & .10 & .69 & .53 \\
\hline Ítem 20 & .08 & .54 & .02 & .01 & -.16 & .15 & .66 & .59 \\
\hline Ítem 24 & -.07 & .72 & .03 & -.03 & -.10 & -.05 & .65 & .65 \\
\hline Ítem 33 & .01 & .39 & .05 & -.13 & .12 & .02 & .68 & .52 \\
\hline Ítem 34 & .01 & .64 & -.13 & .03 & .13 & -.06 & .64 & .55 \\
\hline Ítem 36 & -.08 & .25 & .15 & -.07 & .33 & -.13 & .70 & .43 \\
\hline \multicolumn{9}{|l|}{ Futuro } \\
\hline Ítem 6 & -.16 & .13 & .56 & -.06 & -.01 & -.05 & .60 & .57 \\
\hline Ítem 9 & -.02 & -.11 & .61 & -.011 & .16 & -.22 & .59 & .62 \\
\hline Ítem 13 & .12 & -.04 & .42 & .07 & -.03 & -.02 & .62 & .53 \\
\hline Ítem 16 & -.03 & .01 & .48 & .08 & -.07 & .08 & .61 & .52 \\
\hline Ítem 18 (*) & .14 & .28 & .27 & .04 & -.28 & -.29 & .65 & .55 \\
\hline Ítem 23 & -.02 & -.06 & .48 & -.13 & -.22 & .19 & .61 & .54 \\
\hline Ítem 32 & -.07 & .01 & .55 & .13 & .06 & -.14 & .60 & .58 \\
\hline Ítem $41(*)$ & -.05 & -.04 & .14 & -.06 & -.06 & -.41 & .67 & .39 \\
\hline \multicolumn{9}{|l|}{ Pasado Positivo } \\
\hline Ítem 2 & -.09 & -.03 & .18 & .29 & .25 & .26 & .58 & .57 \\
\hline Ítem 4 & .10 & .05 & -.03 & .43 & -.09 & .07 & .59 & .55 \\
\hline Ítem 7 & -.13 & .10 & .06 & .62 & -.10 & -.10 & .58 & .57 \\
\hline Ítem 11 & .18 & -.06 & .21 & .34 & .12 & .10 & .59 & .55 \\
\hline Ítem 15 & -.02 & .27 & .19 & .41 & .04 & .16 & .57 & .61 \\
\hline Ítem 19 (*) & -.16 & -.05 & -.16 & .54 & .53 & -.09 & .63 & .43 \\
\hline Ítem 22 & .25 & -.07 & .24 & .11 & -.05 & .27 & .62 & .45 \\
\hline Ítem 37 & .03 & -.07 & .18 & .37 & .24 & -.06 & .60 & .51 \\
\hline \multicolumn{9}{|l|}{ Presente Fatalista } \\
\hline Ítem 10 & -.06 & -.05 & -.04 & -.16 & .49 & .30 & .63 & .58 \\
\hline Ítem 27 & -.01 & .23 & -.04 & -.18 & .10 & .19 & .65 & .44 \\
\hline Ítem 29 & .13 & .07 & -.11 & .09 & .49 & .02 & .62 & .62 \\
\hline Ítem 30 & .29 & .02 & .02 & .02 & .44 & -.08 & .61 & .62 \\
\hline Ítem 31 & -.13 & -.02 & .03 & -.17 & .61 & .11 & .62 & .61 \\
\hline Ítem 35 & .48 & -.22 & -.07 & .18 & .22 & -.02 & .65 & .53 \\
\hline Ítem 39 & .02 & -.05 & -.07 & -.11 & .21 & .44 & .66 & .51 \\
\hline $\begin{array}{l}\text { Porcentaje de la } \\
\text { Varianza explicada }\end{array}$ & $13.57 \%$ & $8.47 \%$ & $6.92 \%$ & $5.18 \%$ & $4.19 \%$ & $3.76 \%$ & & \\
\hline Alfa de Cronbach & .80 & .70 & .65 & .63 & .67 & & & \\
\hline
\end{tabular}

Nota. $(*)=$ ítems inversos.

Los números en negrita son los pesos factoriales del factor predominante.

fueron en la mayoría de los casos \pm 1 , lo cual denota una distribución normal y cumple el primer supuesto para la realización de un análisis factorial. De acuerdo con los criterios de Martínez-Arias (1995) respecto de la cantidad de observaciones necesarias para realizar análisis 
factorial, la cantidad de casos incluidos en este estudio $(N=343)$ resulta suficiente para un análisis básico. Se evaluó la composición de factores considerando conveniente explorarlos sin tener en cuenta a priori la estructura de cinco factores encontrada en la muestra anterior y en la versión original (Pérez-Gil, Chacón, \& Moreno, 2000). Así, se realizó un análisis paralelo clásico para determinar el número de factores (Horn, 1965). Este análisis arrojó una estructura de seis factores que explicaron el $43 \%$ de la varianza que fueron extraídos por el método ULS recomendado para estos casos (Forero, Maydeu-Olivares, \& Gallardo-Pujol, 2009).

A partir de esto se procedió a hacer el análisis factorial exploratorio (AFE) con los seis factores que sugirió el análisis paralelo. Este fue el primer modelo puesto a prueba. Se utilizó la rotación Promax para mejorar la interpretación de los resultados (Fabrigar, Wegener, MacCallum, \& Strahan, 1999). Promax primero realiza una rotación Varimax y luego una rotación oblicua para aumentar la adaptación a una estructura simple (Russell, 2002). La significación del Test de Esfericidad de Bartlett $\left(\mathrm{x}^{2}{ }_{(820)}=3640.4 ; p<.001\right)$ fue significativo y el índice de adecuación muestral Kaiser-Meyer-Olkin (KMO) indicó una adecuación de .78 , lo que sugiere una buena correlación entre los ítems y una buena adecuación muestral respectivamente, evidenciando la pertinencia de un análisis factorial (Kaiser \& Raice, 1974). Los seis primeros factores explicaron el $42 \%$ de la varianza $(13.57 \%, 8.47 \%, 6.92 \%, 5.18 \%, 4.19 \%$ y $3.76 \%)$.

El programa FACTOR realiza un análisis factorial semiconfirmatorio, por lo cual arroja medidas de índice de bondad de ajuste (LorenzoSeva \& Ferrando, 2013). Las medidas para probar el modelo aceptadas como medidas de ajuste robustas son el índice de bondad de ajuste (GFI) y el índice ajustado de bondad de ajuste (AGFI), los cuales son independientes del tamaño muestral; y el índice de ajuste comparado (CFI) (Hoyle, 1995). Siguiendo las recomendaciones de $\mathrm{Hu}$ y Bentler (1999) los criterios para decidir la bondad de ajuste de un determinado modelo son: GFI >.90; AGFI >.90; CFI >.95; SRMS<.08. En este caso el modelo $\left(\mathrm{X}^{2}(589)=458.970 ; \quad P=.99\right)$ proporcionó un ajuste excelente a los datos: $\mathrm{GFI}=.96 ; \mathrm{AGFI}=.95 ; \mathrm{CFI}=1 ; \mathrm{RMSR}=.04$.
Nuevamente, se siguieron los criterios sugeridos por Marsh, Martin y Jackson (2010) y se analizó la matriz de correlaciones para pesquisar qué ítems podrían ser eliminados para llegar a la versión final del ZTPI-B. Luego, se observó la correlación ítem factor y el valor de alfa de Cronbach si se elimina el elemento y, finalmente, se realizó un análisis racional teniendo en cuenta los postulados del modelo de Zimbardo- y se cotejaron las definiciones de cada marco temporal con los ítems que resultaron dudosos o malos psicométricamente. A partir de la conjunción de estos análisis se decidió la eliminación de los ítems 1, 2, 8, 17, 18, 22, 25, 27, $35,36,39$ y 41 . Cabe destacar que si bien en el AFE se forzaron los seis factores sugeridos por el análisis paralelo, el sexto factor incluyó solo tres ítems, de los cuales dos ítems tuvieron carga compartida y uno tuvo carga negativa, es decir que este último factor no es significativo y puede ser eliminado (ver Tabla 2).

El modelo de 29 ítems se puso a prueba y fue sometido a un análisis factorial exploratorio utilizando el método de extracción ULS y rotación Promax, considerando ahora cinco factores. La significación del Test de Esfericidad de Bartlett $\left(\chi^{2}(406)=2375 ; p<.001\right)$ fue significativo y el índice de adecuación muestral Kaiser-MeyerOlkin (KMO) indicó una adecuación de .78. Los cinco primeros factores explicaron el $45.46 \%$ de la varianza $(15.3 \%, 10.25 \%, 8.59 \%, 5.94 \%$ y $5.35 \%)$. El porcentaje de varianza explicado es mayor al 36\% obtenido por Zimbardo y Boyd (1999). El modelo $\left(\chi^{2}(271)=219.784 ; \quad P=.98\right)$ proporcionó un ajuste excelente a los datos: GFI=.97; AGFI=.96; $\quad \mathrm{CFI}=1 ; \quad \mathrm{SRMS}=0.03 . \quad \mathrm{El}$ análisis de la matriz de correlaciones mostró que todas las cargas fueron superiores a .35 .

\section{Estudio 2}

Se efectuó un segundo estudio con el fin de realizar un análisis factorial semiconfirmatorio para contrastar el modelo original de los cinco factores propuesto por Zimbardo y Boyd (1999) con la abreviación de la escala que se propone en el estudio anterior.

\section{Participantes}

La muestra estuvo compuesta por 272 personas, $65 \%$ mujeres, todos de nacionalidad 
argentina. Las edades estuvieron comprendidas entre 18 y 65 años $(M E=33.4 ; D E=13.6)$. Todos los participantes viven en zonas urbanas de Buenos Aires, 55\% en Gran Buenos Aires y 45\% en la Ciudad Autónoma de Buenos Aires. En cuanto al nivel de estudios alcanzados $42 \%$ tiene terciario/universitario incompleto, $28 \%$ terciario/universitario completo, $25 \%$ secundario completo y $5 \%$ posgrado/doctorado. Participaron del estudio entre marzo y octubre de 2019.

\section{Instrumentos}

Datos sociodemográficos. Se utilizó el mismo cuestionario que en el estudio anterior.

Inventario de Perspectiva Temporal de Zimbardo Breve (ZTPI-B). Para este estudio se utilizó la versión de 29 ítems realizada a partir del análisis informado en el estudio anterior. Los ítems evalúan los cinco factores propuestos por los autores originales: pasado negativo (7 ítems), presente hedonista (6 ítems), futuro (6 ítems), pasado positivo (6 ítems) y presente fatalista (4 ítems).

Escala de Malestar Psicológico de Kessler $K$ 10. Se utilizó la misma escala que en el estudio anterior.

Escala de Autocontrol (EAC). Se utilizó la misma escala que en el estudio anterior.

\section{Procedimiento}

Los participantes aceptaron el consentimiento informado correspondiente donde se les garantizó la confidencialidad de los datos que se obtuvieran. Luego se aplicaron los instrumentos en el siguiente orden: cuestionario de datos sociodemográficos, ZTPI-B, K-10, EAC. Los cuestionarios fueron auto-administrados y no hubo compensación económica o de otro tipo para los participantes.

\section{Análisis de datos}

Para realizar el análisis factorial semiconfirmatorio se utilizó el programa estadístico FACTOR 9.2 (Lorenzo-Seva \& Ferrando, 2013). Luego, con el programa IBM SPSS 25 , se calculó la prueba $t$ para muestras independientes para analizar las diferencias según sexo y edad, y se realizaron análisis de correlación con el coeficiente $r$ de Pearson para evaluar la validez externa del instrumento.

\section{Resultados}

La muestra $(N=272)$ alcanzó el tamaño sugerido por algunos autores como mínimo necesario para llevar a cabo un análisis factorial semiconfirmatorio ( $N=200$ ) (Jackson, 2003; Kline, 2005). En cuanto a la normalidad multivariante, el Coeficiente de Mardia para la asimetría no fue significativo $(M=165.311 ; p=1)$, mientras que para la curtosis fue significativo $(M=1017.998$; $p<.001)$ lo cual se comparece con no normalidad multivariada (Mardia, 1970).

Se empleó el método de estimación ULS y rotación Promax. La significación del Test de Esfericidad de Bartlett $\left(\mathrm{x}^{2}(406)=2423 ; p<.001\right)$ fue significativo y el índice de adecuación muestral Kaiser-Meyer-Olkin (KMO) indicó una adecuación de .79. Los cinco primeros factores explicaron el $48.87 \%$ de la varianza (18\%, $12.13 \%, 7.33 \%, 5.98 \%$ y $5.41 \%)$. Se obtuvieron los siguientes resultados: $X^{2}(271)=196.074$; $p=.99 ; \mathrm{NNFI}=1 ; \mathrm{CFI}=1 ; \mathrm{GFI}=0.97 ; \mathrm{AGFI}=0.95$; $\mathrm{RMSR}=.04$.

Se calculó el coeficiente alfa de Cronbach para cada escala. Para el pasado negativo se obtuvo un valor de .84; pasado positivo .60; presente hedonista .70; futuro .65; y presente fatalista .69 .

Respecto de las diferencias en función del sexo solamente se hallaron diferencias estadísticamente significativas en el factor futuro $\left(t_{(247)}=3.04 ; p<.05\right)$, siendo mayor la media en las mujeres $(M E=4.01 ; D E=.52)$ que en los hombres $(M E=3.77 ; D E=.70)$. Respecto de la edad se dividió a los participantes en dos grupos: jóvenes $(<30)$ y adultos $(\geq 30)$. Se encontraron diferencias significativas en tres de los cinco factores. En el pasado negativo $\left(t_{(247)}=-4.19 ; p<.05\right)$ los jóvenes presentan una media significativamente mayor $(M E=2.93 ; \quad D E=.89)$ que la de los adultos $(M E=2.47 ; D E=.81)$. En el presente hedonista $\left(t_{(247)}=-3.67 ; p<.05\right)$ la media es significativamente mayor en los jóvenes $(M E=3.15 ; D E=.66)$ que en los adultos $(M E=2.83 ; D E=.70)$. En el factor pasado positivo $\left(t_{(247)}=-2.63 ; p<.05\right)$ los jóvenes presentan una media significativamente mayor $(M E=3.75 ; \quad D E=.62)$ que la de los adultos $(M E=3.54 ; D E=.60)$.

Respecto de la validez externa, se encontraron correlaciones estadísticamente significativas entre 
Tabla 3. Cargas factoriales del ZTPI-B

\begin{tabular}{|c|c|c|c|c|c|}
\hline \multirow[t]{2}{*}{ Ítems* } & \multicolumn{5}{|c|}{ Factores** } \\
\hline & $\begin{array}{c}\text { Pasado } \\
\text { Negativo }\end{array}$ & $\begin{array}{l}\text { Presente } \\
\text { Hedonista }\end{array}$ & Futuro & $\begin{array}{c}\text { Pasado } \\
\text { Positivo }\end{array}$ & $\begin{array}{l}\text { Presente } \\
\text { Fatalista }\end{array}$ \\
\hline Ítem 1 (3) & .56 & .01 & -.16 & .12 & -.01 \\
\hline Ítem 9 (12) & .69 & .00 & -.01 & -.00 & .06 \\
\hline Ítem $16(21)$ & .50 & -.05 & .03 & .04 & .02 \\
\hline Ítem 19 (26) & .75 & .10 & .22 & -.24 & .00 \\
\hline Ítem 20 (28) & .68 & .01 & .05 & .19 & -.02 \\
\hline Ítem 25 (38) & .86 & .03 & .06 & -.03 & -.11 \\
\hline Ítem 29 (40) & .58 & -.09 & -.04 & .16 & .08 \\
\hline Ítem $3(5)$ & .05 & .13 & -.27 & .34 & .12 \\
\hline Ítem 11 (14) & -.04 & .31 & .20 & .15 & .10 \\
\hline Ítem 15 (20) & .26 & .63 & -.04 & .05 & -.23 \\
\hline Ítem 18 (24) & -.10 & .84 & .11 & -.14 & .06 \\
\hline Ítem 21 (33) & -.03 & .76 & -.08 & -.09 & .70 \\
\hline Ítem 27 (34) & .06 & .37 & -.27 & .22 & .08 \\
\hline Ítem 4 (6) & -.16 & .02 & .43 & .19 & .16 \\
\hline Ítem 6 (9) & .11 & -.10 & .45 & .09 & .01 \\
\hline Ítem 10 (13) & .14 & -.06 & .41 & .20 & .03 \\
\hline Ítem 13 (16) & -.00 & .09 & .45 & .27 & -.00 \\
\hline Ítem 17 (23) & .19 & -.00 & .63 & -.05 & -.06 \\
\hline Ítem 26 (32) & -.12 & .07 & .58 & .02 & .15 \\
\hline Ítem 2 (4) & .29 & -.12 & .00 & .49 & -.01 \\
\hline Ítem 5 (7) & -.26 & .14 & .19 & .41 & -.15 \\
\hline Ítem 8 (11) & .30 & -.12 & .11 & .47 & .21 \\
\hline Ítem 12 (15) & .06 & .06 & .17 & .53 & -.04 \\
\hline Ítem $14(*)(19)$ & -.26 & -.06 & -.03 & .34 & -.25 \\
\hline Ítem 28 (37) & .00 & -.04 & .06 & .44 & -.04 \\
\hline Ítem 7 (10) & -.02 & .00 & .05 & .07 & .56 \\
\hline Ítem 22 (29) & .10 & .13 & -.04 & .07 & .53 \\
\hline Ítem 23 (30) & .20 & -.07 & -.06 & .05 & .47 \\
\hline Ítem 24 (31) & -.06 & .01 & .10 & -.06 & .78 \\
\hline Porcentaje de & & & & & \\
\hline la Varianza & $12.13 \%$ & $5.98 \%$ & $7.33 \%$ & $5.98 \%$ & $18 \%$ \\
\hline Explicada & & & & & \\
\hline Alfa de Cronbach & .84 & .70 & .65 & .60 & .69 \\
\hline
\end{tabular}

Nota. $(*)=$ ítem inverso

*Los números entre paréntesis corresponden al número de ítem originario.

**Los números en negrita son los pesos factoriales del factor predominante.

el autocontrol y cuatro de los cinco factores del ZTPI-B: con futuro $(r=.41 ; p<.005)$, con pasado negativo $(r=-.37 ; p<.005)$, con presente fatalista $(r=-.31, p<.005)$ y con presente hedonista $(r=.14$; $p<.005)$. En relación a la escala de malestar psicológico, se encontraron correlaciones estadísticamente significativas con tres de los cinco factores: con pasado negativo ( $r=.57$; $p<.005)$, con presente fatalista $(r=.23 ; p<.005) \mathrm{y}$ con presente hedonista $(r=.21 ; p<.005)$. De acuerdo con los criterios de Cohen (1988), los coeficientes pueden considerarse con un tamaño del efecto grande entre malestar psicológico y pasado negativo, moderado entre autocontrol y futuro y autocontrol y pasado negativo y pequeño en las demás.

\section{Discusión y Conclusiones}

El presente artículo tuvo como objetivo presentar una versión abreviada del Inventario de Perspectiva Temporal de Zimbardo para Buenos Aires. Para ello se realizaron dos estudios complementarios. En el primero se partió de un análisis paralelo (Horn, 1965) y luego se pusieron a prueba dos modelos usando un análisis factorial exploratorio con extracción ULS y rotación Promax. Estos análisis están recomendados para estos casos y han demostrado ser más eficaces que los utilizados por los autores originales (Fabrigar et al., 1999; Forero et al., 2009). El análisis de las cargas factoriales de la matriz de correlaciones y los índices de bondad de ajuste mostraron que el modelo de 29 ítems es más satisfactorio que el de partida ya que los índices mejoran, el porcentaje 
de varianza explicada aumenta, las cargas factoriales son aceptables con muy escasas cargas compartidas y su contenido es concordante teóricamente con las ideas de Zimbardo y Boyd (1999) acerca de la perspectiva temporal.

En el segundo estudio se realizó un análisis factorial semiconfirmatorio para confirmar la estructura del ZTPI-B y contrastarla con el modelo original lo cual es recomendado cuando existen estudios previos (Cupani, 2012). También se realizaron análisis de validez externa y se evaluaron las diferencias por edad y sexo, todos análisis sugeridos en las buenas prácticas de la realización, adaptación y/o abreviación de instrumentos de medición. Si bien en este estudio los datos del análisis factorial semiconfirmatorio señalan la bondad de ajuste al modelo teórico, no obstante antecedentes de abreviaciones del ZTPI presentaron índices no satisfactorios o realizaron modificaciones importantes de la prueba para lograr un buen ajuste del modelo (Apostolidis \& Fieulaine, 2004; González Lomelí et al., 2017; Wakefield et al., 2010; Zimbardo \& Boyd, 1999). Esto podría señalar limitaciones en cuanto a la universalidad de la estructura interna que deberían ser estudiadas en profundidad en futuras investigaciones de carácter transcultural.

Por otro lado, el nivel de confiabilidad de cada dimensión del ZTPI-B medido a través del alfa de Cronbach (.60 a .84), se encuentra dentro del rango reportado en estudios previos del ZTPI en idioma español (ver Tabla 1) que lo sitúan entre .59 y .80, y coincide también con el observado en diversas versiones abreviadas en otros idiomas (Sircova et al., 2014; Przepiorka, Sobol-Kwapinska \& Jankowski, 2016). Respecto a estos valores cabe mencionar que el valor de .60 es considerado como adecuado para estudios en ciencias sociales $\mathrm{o}$ en etapas iniciales de investigación (Nunnally, 1994; Nieva \& Sorra, 2003). Otros consideran que ese valor es apropiado en estudios de prueba de instrumentos (Wensing, Elwyn, Edwards, Vingerhoets, \& Grol, 2002). Esto muestra que, en nuestro contexto, se obtuvieron evidencias satisfactorias de fiabilidad y nos permite saber que las mediciones con esta escala no estarán sesgadas por el método seguido.

Respecto a las evidencias de validez externa en primer lugar, las correlaciones positivas entre pasado negativo y presente fatalista con malestar psicológico y negativa con autocontrol ratifican el significado de estos factores. El pasado negativo refiere a una actitud negativa y pesimista hacia el pasado y el presente fatalista supone una actitud de indefensión con respecto a la vida en general (Zimbardo \& Boyd, 1999). A la vez, la asociación positiva con el malestar psicológico indica que estas actitudes se vinculan con un abanico de síntomas vinculados a la depresión y la ansiedad. Finalmente, estos resultados van en línea con lo hallado en diversos estudios que muestran que el pasado negativo y presente fatalista aparecen vinculados negativamente con bienestar psicológico y se relacionan negativamente con la salud (Oyanadel \& Buela-Casal, 2010; Sircova et al., 2014; Brenlla et al., 2019).

En segundo lugar, se halló una correlación significativa negativa entre presente hedonista y autocontrol, mientras que con el malestar psicológico la correlación fue positiva. Esta dimensión refiere a una actitud de disfrute del momento actual sin evaluar las consecuencias; y una positiva con el futuro, entendido como la tendencia a planificar metas a largo plazo. Las investigaciones demuestran que las personas con puntajes elevados de autocontrol presentan un mejor ajuste psicológico, mejores habilidades interpersonales y menos problemas de comportamiento tales como los vinculados al consumo excesivo de drogas, alcohol o comida (Tangney et al., 2004; Garrido et al., 2017; Garrido et al., 2018). Asimismo, Sircova et al. (2014) señalan que el futuro se asocia con bienestar psicológico, mientras que el presente hedonista se relaciona con comportamientos de riesgo.

En relación con las diferencias halladas en función del sexo y edad los resultados muestran que la variabilidad en las puntuaciones del ZTPIB se deben en parte a la edad y secundariamente al sexo. Solamente se encontraron diferencias estadísticamente significativas en el factor futuro, siendo la media fue mayor en las mujeres, lo cual coincide con estudios previos (Díaz-Morales, 2006; Brenlla et al., 2019). Respecto a la edad, en el presente hedonista la media es significativamente mayor en los jóvenes. Esto concuerda con estudios de diversas culturas que muestran que los jóvenes tienen una preeminencia de orientación al presente y que, a medida que 
aumenta la edad, la orientación temporal tiende a tener una preferencia marcada por el futuro (Brenlla, Willis, \& Germano, 2016; D'Alessio, Guarino, De Pascalis, \& Zimbardo, 2003; Díaz Morales, 2006; Germano \& Brenlla, 2018; Sircova et al, 2014; Zimbardo \& Boyd, 1999).

Respecto a las diversas versiones abreviadas del ZTPI, tal como se mencionó previamente, existen diferencias en la cantidad de ítems del cuestionario, de ítems para cada factor y de dimensiones que evalúa el inventario. Estas variaciones se deben, entre otras cuestiones, al idioma, la cultura, la muestra con la cual se hizo cada uno de los estudios y los análisis de datos respectivos. De manera global, puede decirse que las versiones presentan entre 15 y 36 ítems; como mínimo tres ítems por factor (para una revisión más exhaustiva ver Przepiorka, et al., 2016). Una de las dos versiones abreviadas que existen para población estadounidense evalúa solamente los factores futuro y pasado (Keough, Zimbardo, \& Boyd, 1999) y en las versiones italiana y polaca se eliminaron algunas de las dimensiones por presentar baja consistencia interna (D'Alessio et al., 2003; Przepiorka, et al., 2016). El cuestionario que aquí se presenta está compuesto por 29 ítems, entre cuatro y siete ítems por factor, y se conservan las cinco dimensiones originales, cada una con buenos índices de fiabilidad.

Zimbardo y Boyd (1999) fueron pioneros en introducir la evaluación integral de la orientación temporal. Si bien existen otros instrumentos para la evaluación del tiempo psicológico, estos son referidos al futuro. Entre ellos, el Método de Inducción Motivacional (Nuttin \& Lens, 1985), la Escala de Consideración de las Consecuencias Futuras (Strathman, Gleicher, Boninger, \& Edwards, 1994) y la Escala de Ansiedad Futura (Zaleski, 1996). Por su parte, el ZTPI fue diseñado para evaluar distintos aspectos vinculados a la temporalidad, pone el foco en cinco dimensiones del tiempo y en esto reside su valor. Recientemente se desarrolló una prueba que incluye también la dimensión futuro negativo, que se recomienda para futuras investigaciones (Ortuño, Janeiro, Paixão, Esteves, \& Cordeiro, 2017).

De esta manera, al momento de evaluar temporalidad de forma global, el ZTPI es la prueba más utilizada lo cual coadyuva a justificar el porqué de contar con este instrumento adaptado para nuestro medio. En la actualidad contamos con una versión completa del ZTPI para adolescentes (Galarraga \& Stover, 2016), una para adultos (Brenlla et al, 2019) y a estas sumamos la versión abreviada. Es importante señalar que tener una prueba estandarizada y fácil de administrar para medir la perspectiva temporal puede ser valioso para el área clínica ya que la perspectiva temporal balanceada se encuentra asociada positivamente a la salud y es posible de ser modificada con éxito, por lo tanto, puede ser incluida como una variable terapéutica (Oyandel et al., 2014; Sircova et al., 2014). Además, el presente artículo permite profundizar en la discusión sobre la importancia de adaptar los instrumentos a las características de las poblaciones objetivo.

No obstante, todo estudio tiene sus limitaciones y este no es la excepción. Primero, los participantes fueron todos residentes de zonas urbanas de Buenos Aires y esto no es representativo de la población argentina. Para futuros estudios sería conveniente ampliar la muestra para generar un instrumento válido y fiable para todo el país. Segundo, no se realizó un estudio test-retest; este método es de importancia para neutralizar el efecto de aprendizaje o memoria en la respuesta a un instrumento de medición y, en forma aproximada, analizar la estabilidad de las puntuaciones acerca de los rasgos medidos a través del tiempo. Tercero, habría que continuar validando la estructura factorial con estudios factoriales confirmatorios con tamaños muestrales más grandes y representativos de la población objeto de estudio. Se sugiere también promover el estudio transcultural de las abreviaciones del ZTPI realizadas para conocer si en todos los casos se replica el modelo teórico propuesto por los autores. Se recomienda incluir en un futuro estudio una proporción más significativa de personas mayores a 40 años para lograr una distribución más homogénea que la de este estudio. Con un mayor tamaño muestral se podría además evaluar mediante un análisis factorial confirmatorio multigrupo la propiedad de invarianza del instrumento en función de la edad. Por último, a la luz de los resultados informados en este artículo, es recomendable investigar una 
versión de 27 o 28 ítems ya que hubo dos ítems que podrían ser eliminados o modificados para obtener una estructura factorial más clara. Se trata del ítem 3 por no cargar en el factor que se esperaba y el 14 que es el único inverso y además su carga factorial no es alta, este último podría o bien eliminarse o bien redactarse de forma directa.

En suma, los resultados descritos en este artículo avalan la utilización del ZTPI-B en tareas de investigación para la evaluación de población urbana de Buenos Aires. En este sentido, hay que decir que los análisis de las puntuaciones obtenidas indican evidencias satisfactorias de consistencia interna, validez de contenido, de criterio externo y de constructo.

Entre las futuras líneas de investigación se encuentran las asociadas a los estudios de tiempo psicológico en adultos. Este artículo muestra que las diferentes dimensiones de la orientación temporal se asocian de diversas formas a la capacidad de autocontrol y al malestar psicológico. Por ende, la evaluación de la perspectiva temporal podría ser utilizada en el ámbito clínico. Por otro lado, las investigaciones que tratan el tópico de tiempo incluyen la perspectiva temporal como un constructo fundamental que organiza la conducta de las personas, lo cual tiene consecuencias en su toma de decisiones. En este sentido, el ZTPI-B puede ser utilizado para evaluar aspectos motivacionales y ser incluido en las áreas de investigación y aplicación tales como orientación vocacional y ocupacional. Por otro lado, las evidencias de diferencias en la perspectiva temporal respecto de la edad pueden ser un aporte significativo para la psicología del desarrollo. Ampliar esta línea de investigación podría ayudar a comprender mejor las diferencias en las distintas etapas de la vida respecto de la conceptualización del tiempo, que es un aspecto fundamental en las teorías del desarrollo actuales (Carstensen, 2006).

Finalmente, este estudio se enfoca en las diferencias individuales respecto de la perspectiva temporal, pero sería también interesante estudiar este concepto a nivel inter-cultural e incluso realizar un estudio longitudinal para evaluar diferencias entre generaciones, ya que podría aportar sustento empírico a las teorías posmodernistas sobre las sociedades actuales en las cuales hay un mayor anclaje en el presente debido a la velocidad de los cambios tecnológicos, la supremacía del consumo y la globalización (Bauman, 2015). Otra posible línea de investigación sería el estudio de las diferencias en perspectiva temporal entre personas que viven en zonas urbanas y aquellas de zonas rurales. Puede observarse entonces los potenciales usos del ZTPI-B tanto en psicología aplicada e investigación.

\section{Referencias}

Apostolidis, $\quad$ T., $\quad \& \quad$ Fieulaine, N. (2004). Validación francesa de la escala de temporalidad. European Journal of Applied Psychology, 54 (3), 207-217.

Bauman, Z. (2015). Modernidad líquida. México: Fondo de cultura económica.

Brenlla, M. E., \& Aranguren, M. (2010). Adaptación argentina de la Escala de Malestar Psicológico de Kessler (K10). Revista de Psicología, 28(2), 308-340. Recuperado de http://revistas.pucp.edu.pe/

Brenlla, M. E., Willis, B., \& Germano, G. (2016). Estimación del tiempo y perspectiva temporal en distintas etapas de la adultez. Investigaciones en Psicología, 26(1), 27-34. Recuperado de http://www.psi.uba.ar/investigaciones.php?var =investigaciones/revistas/investigaciones/inve stigaciones.php

Brenlla, M. E., Germano, G., \& Zapater, J. (2019). Adaptación linguística, estructura factorial y fiabilidad del Inventario de Perspectiva Temporal de Zimbardo para Buenos Aires. Interdisciplinaria. En prensa.

Carstensen, L. L. (2006). The influence of a sense of time on human development. Science, 312(5782), 1913-1915. http://DOI:10.1126/science.1127488

Cohen, J. (1988). Statistical power analysis for the behavioral sciences (2nd edition). Hillsdale, NJ: Erlbaum.

Corral-Verdugo, V., Fraijo-Sing, B., \& Pinheiro, J. Q. (2006). Sustainable behavior and time perspective: Present, past, and future orientations and their relationship with water conservation behavior. Interamerican Journal of Psychology, 40(2), 139-147. Recuperado de http://pepsic.bvsalud.org/ 
Cupani, M. (2012). Análisis de Ecuaciones Estructurales: conceptos, etapas de desarrollo y un ejemplo de aplicación. Revista Tesis, 1(1), 186-199. Recuperado de https://www.researchgate.net/profile/Marcos_ Cupani/publication

D'Alessio, M., Guarino, A., de Pascalis, V., \& Zimbardo, P. G. (2003). Testing Zimbardo's Stanford Time Perspective Inventory (STPI)Short form: An Italian study. Time \& Society, 12(2),

333-347. http://dx.doi.org/10.1177/0961463X030122010

Díaz-Morales, J. F. (2006). Estructura factorial y fiabilidad del Inventario de Perspectiva Temporal de Zimbardo. Psicothema, 18(3), 565-571. Recuperado de http://www.psicothema.com

Fabrigar, L. R., Wegener, D. T., MacCallum, R. C., \& Strahan, E. J. (1999). Evaluating the use of exploratory factor analysis in psychological research. Psychological methods, 4(3), 272. https://doi.org/10.1037/1082-989X.4.3.272

Forero, C. G., Maydeu-Olivares, A., \& GallardoPujol, D. (2009). Factor analysis with ordinal indicators: A Monte Carlo study comparing DWLS and ULS estimation. Structural Equation Modeling, 16(4), 625-641. doi: https://doi.org/10.33588/rn.4804.2008084

Galarraga, M. L., \& Stover, J. B. (2016). Inventario de Perspectiva Temporal de Zimbardo: Adaptación en estudiantes de nivel medio de Buenos Aires. Psicodebate. Psicología, Cultura y Sociedad, 16(1), 109128. http://dx.doi.org/10.18682/pd.v16i1.540

Garrido, S., Cupani, M., \& Arbach, K. (2017). Aplicación del Modelo de Escala de Clasificación Para Examinar las Propiedades Psicométricas de la Self-Control Scale. Psykhe, 26(2), 1-12. https://doi.org/10.7764/psykhe.26.2.980

Garrido, S. J., Morán, V., Azpilicueta, A. E., Cortez, F., Arbach, K., \& Cupani, M. (2018). Análisis de modelos rivales unidimensionales y bidimensionales de la escala breve de autocontrol en estudiantes universitarios argentinos. Psicodebate. Psicología, Cultura y Sociedad, 18(2), 26-37. http://dx.doi.org/10.18682/pd.v18i2.745

Germano, G., \& Brenlla, M. E. (2018). Temporalidad subjetiva en jóvenes de buenos aires. Trabajo presentado en $\mathrm{X}$ Congreso Internacional de Investigación y Práctica Profesional en Psicología, Buenos Aires. Recuperado de http://jimemorias.psi.uba.ar/index.aspx?anio= 2018

González-Lomelí, D. G., Maytorena-Noriega M. Á., Cárdenas-Niño, L., \& Tapia-Fonllem C. O. (2018). Perspectiva temporal de estudiantes universitarios mexicanos y colombianos. Revista Iberoamericana de Diagnóstico y Evaluación - e Avaliação Psicológica, 1(46), 133-145. https://doi.org/10.21865/RIDEP46.1.10

Herrero, J. (2010). El análisis factorial confirmatorio en el estudio de la estructura y estabilidad de los instrumentos de evaluación: Un ejemplo con el Cuestionario de Autoestima CA-14. Psychosocial Intervention, 19(3), 289-300. https://doi.org/10.5093/in2010v19n3a9

Horn, J. L. (1965). A rationale and test for the number of factors in factor analysis. Psychometrika, $\quad 30(2), \quad$ 179-185. https://doi.org/10.1007/BF02289447

Hoyle, R. H. (1995). Structural equation modeling: Concepts, issues, and applications. London: Sage.

Hu, L. T., \& Bentler, P. M. (1999). Cutoff criteria for fit indexes in covariance structure analysis: Conventional criteria versus new alternatives. Structural equation modeling: a multidisciplinary journal, 6(1), 1-55. https://doi.org/10.1080/10705519909540118

Jackson, D. L. (2003). Revisiting sample size and number of parameter estimates: Some support for the N:q hypothesis. Structural Equation Modeling, 10(1), 128-141. doi:10.1207/S153 28007SEM1001_6

Kaiser, H. F., \& Rice, J. (1974). Little jiffy, mark IV. Educational and Psychological Measurement, $\quad 34(1)$, 111-117. https://doi.org/10.1177/001316447403400115

Keough, K. A., Zimbardo, P. G., \& Boyd, J. N. (1999). Who's smoking, drinking, and using drugs? Time perspective as a predictor of substance use. Basic and Applied Social Psychology, 21(2), 149-164. http://dx.doi.org/10.1207/S15324834BA2102 07 
Kessler, R., \& Mroczek, D. (1994). Final version of our non-specific Psychological Distress Scale. Ann Arbor, MI: Survey Research Center of the Institute for Social Research, University of Michigan.

Kline, R. B. (2005). Principles and practices of structural equation modeling. New York, NY: Guilford.

Klingeman, H. (2001). The time game: Temporal perspectives of patients and staff in alcohol and drug treatment. Time \& Society, 10(2), 303-328. http://dx.doi.org/10.1007/s11031-006-9018-9

Lewin, K. (1942). Time perspective and morale. En Lewin, G. (Ed.) Resolving Social Conflicts (pp 103-124). New York: Harper.

Lorenzo-Seva, U., \& Ferrando, P. J. (2013). FACTOR 9.2 A comprehensive program for fitting exploratory and semiconfirmatory factor analysis and IRT Models. Applied Psychological Measurement, 37(6), 497-498. https://doi.org/10.1177/0146621613487794

Mardia, K. V. (1970). Measures of multivariate skewness and kurtosis with applications. Biometrika, 57, 519-530.

Marsh, H. W., Martin, A. J., \& Jackson, S. (2010). Introducing a short version of the physical self-description questionnaire: New strategies, short-form evaluative criteria, and applications of factor analysis. Journal of Sport \& Exercise Psychology, 32(4), 438-482. http://dx.doi.org/10.11588/ijodr.2014.1.12330

Martínez-Arias, R. (1995). Psicometría: Teoría de los test psicológicos y educativos. Madrid: Síntesis.

Montero, I., \& León, O. (2007). A guide for naming research studies in psychology. International Journal of Clinical and Health Psychology, 7, 847-862. Disponible en: http://www.redalyc.org/articulo.oa?id=33770318

Nieva, V. F., \& Sorra, J. (2003). Safety culture assessment: A tool for improving patient safety in healthcare organizations. $B M J$ Quality \& Safety, 12(2), 17-23. http://dx.doi.org/10.1136/qhc.12.suppl_2.ii17

Nunnally, J. C. (1994). Psychometric theory 3E. New York: McGraw-Hill Education.

Nuttin, J., \& Lens, W. (1985). Future time perspective and motivation: Theory and research method. New York: Psychology Press.

Ortuño, V. E., Janeiro, I. N., Paixão, M. P., Esteves, C., \& Cordeiro, P. (2017). Um novo modelo multidimensional da Perspetiva Temporal. Revista Iberoamericana de Diagnóstico y Evaluación -e Avaliação $\quad$ Psicológica, 3(45), 71-84. https://doi.org/10.21865/RIDEP45.3.06

Oyanadel, C., \& Buela-Casal, G. (2010). La percepción del tiempo: Influencias en la salud física y mental. Universitas Psychologica, 10(1), 149-161. https://doi.org/10.11144/Javeriana.upsy101.ptis

Oyanadel, C., Buela-Casal, G., \& Pérez-Fortis, A. (2014). Propiedades Psicométricas del Inventario de Orientación Temporal de Zimbardo en una muestra chilena. Terapia Psicológica, $\quad$ 32(1), 47-55. http://dx.doi.org/10.4067/S071848082014000100005

Oyanadel, C., Buela-Casal, G., Araya, T., Olivares, C., \& Vega, H. (2014). Percepción del tiempo: Resultados de una intervención grupal breve para el cambio del perfil temporal. Suma Psicológica, 1(21), 1-7. https://doi.org/10.1016/S0121-381(14)70001-3

Pérez-Gil, J. A., Moscoso, S. C., \& Rodríguez, R. M. (2000). Validez de constructo: El uso de análisis factorial exploratorio-confirmatorio para obtener evidencias de validez. Psicothema, 12(2), 442-446. Recuperado de http://www.psicothema.es/pdf/601.pdf

Przepiorka, A., Sobol-Kwapinska, M., \& Jankowski, T. (2016). A polish short version of the Zimbardo Time Perspective Inventory. Personality and Individual Differences, 101, 78-89. http://dx.doi.org/10.1016/j.paid.2016.05.047

Russell, D. W. (2002). In search of underlying dimensions: The use (and abuse) of factor analysis in PSPB. Personality and Social Psychology Bulletin, 28(12), 1629-1646. http://dx.doi.org/10.1177/014616702237645.

Sircova, A., van de Vivjer, F. J. R., Osin, E., Milfont, T. L., Feulaine, N., KislaliErginbilgic, A., Zimbardo, P. G., \& 54 miembros del International Time Perspective Research Project (2014). A global look at 
time: A 24-country study of the equivalence of the Zimbardo Time Perspective Inventory. SAGE Open, 4(54), 1-12. https://doi.org/10.1177/2158244013515686

Stolarski, M., Matthews, G., Postek, S., Zimbardo, P. G., \& Bitner, J. (2014). How we feel is a matter of time: Relationships between time perspective and mood. Journal of Happiness Studies, $\quad$ 15(4), 809-827. http://dx.doi.org/10.1007/s10902-013-9450-y

Strathman, A., Gleicher, F., Boninger, D. S., \& Edwards, C. S. (1994). The consideration of future consequences: Weighing immediate and distant outcomes of behavior. Journal of Personality and Social Psychology, 66(4), 742-752.

http://dx.doi.org/10.1037/0022-3514.66.4.742

Tangney, J. P., Baumeister, R. F., \& Boone, A. L. (2004). High self-control predicts good adjustment, less pathology, better grades, and interpersonal success. Journal of Personality, $72(2)$, 271-322. https://doi.org/10.1111/j.00223506.2004.00263.x

Wakefield, C. E., Homewood, J., Taylor, A., Mahmut, M., \& Meiser, B. (2010). Time perspective in hereditary cancer: Psychometric properties of a short form of the Zimbardo Time Perspective Inventory in a community and clinical sample. Genetic testing and molecular biomarkers, 14(5), 617627.

Wensing, M., Elwyn, G., Edwards, A., Vingerhoets, E., \& Grol, R. (2002). Deconstructing patient centred communication and uncovering shared decision making: an observational study. BMC Medical Informatics and Decision Making, 2(1), 2. Disponible en: https://biomedcentral.com/ 1472-6947/2/2

Worthington, R. L., \& Whittaker, T. A. (2006). Scale development research: A content analysis and recommendations for best practices. The Counseling Psychologist, 34(6), 806-838. https://doi.org/10.1177/0011000006288127

Zaleski, Z. (1996). Future anxiety: Concept, measurement, and preliminary research. Personality and Individual
Differences, 21(2), $165-174$ https://doi.org/10.1016/0191-8869(96)00070-0

Zhang, J. W., \& Howell, R. T. (2011). Do time perspectives predict unique variance in life satisfaction beyond personality traits? Personality and Individual Differences, 50(8), 1261-1266. http://dx.doi.org/10.1016/j.paid.2014.11.025.

Zimbardo, P. G., \& Boyd, N. (1999). Putting time in perspective: A valid, reliable, individualdifferences metric. Journal of Personality and Social Psychology, 17(6), 1271-1288. http://dx.doi.org/10.1037/0022-3514.77.6.1271

Zimbardo, P. G., \& Boyd, J. N. (2008). The time paradox. New York: Free Press. 


\section{Anexo I}

\section{Inventario de Perspectiva Temporal de Zimbardo Breve (ZTPI-B)}

Lea cada ítem y responda cada pregunta de la manera más honesta posible: “¿Cuán verdadero con respecto a usted es la afirmación presentada?". Marque la casilla correspondiente usando la siguiente escala: 1=Completamente Falso; 2=Bastante Falso; 3=Neutral; 4=Bastante Verdadero; 5=Completamente Verdadero

\begin{tabular}{|c|c|c|c|c|c|}
\hline & 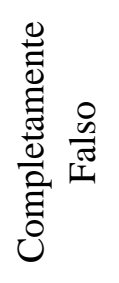 & 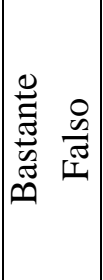 & $\begin{array}{l}\bar{\pi} \\
\stackrel{\Xi}{0} \\
z\end{array}$ & 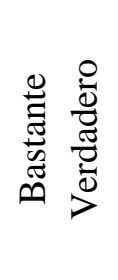 & 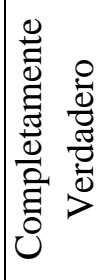 \\
\hline & 1 & 2 & 3 & 4 & 5 \\
\hline 1.A menudo pienso qué debería haber hecho diferente en mi vida. & & & & & \\
\hline 2.Me gusta pensar en mi pasado. & & & & & \\
\hline 3.Hago cosas impulsivamente. & & & & & \\
\hline $\begin{array}{l}\text { 4.Cuando quiero conseguir algo, me fijo metas y pienso en maneras } \\
\text { concretas de conseguirlas. }\end{array}$ & & & & & \\
\hline $\begin{array}{l}\text { 5.Si los pongo en una balanza, tengo muchos más recuerdos buenos } \\
\text { que malos. }\end{array}$ & & & & & \\
\hline $\begin{array}{l}\text { 6.Preparar el trabajo para el día siguiente y cumplir con los plazos es } \\
\text { más importante que la diversión de hoy a la noche. }\end{array}$ & & & & & \\
\hline $\begin{array}{l}\text { 7.Ya que las cosas serán lo que serán, realmente no importa mucho lo } \\
\text { que yo haga. }\end{array}$ & & & & & \\
\hline $\begin{array}{l}\text { 8. Me divierten las historias sobre cómo eran las cosas en los "viejos } \\
\text { tiempos". }\end{array}$ & & & & & \\
\hline 9. Revivo constantemente experiencias dolorosas de mi pasado. & & & & & \\
\hline 10.Me molesta mucho llegar tarde a mis citas y compromisos. & & & & & \\
\hline 11.Idealmente, viviría cada día como si fuese el último. & & & & & \\
\hline $\begin{array}{l}\text { 12.Los recuerdos felices de los buenos tiempos están muy presentes en } \\
\text { mi mente. }\end{array}$ & & & & & \\
\hline 13.Cumplo con las obligaciones para con mis amigos y jefes a tiempo. & & & & & \\
\hline $\begin{array}{l}\text { 14.El pasado tiene tantos recuerdos desagradables que prefiero no } \\
\text { pensar en ellos. }\end{array}$ & & & & & \\
\hline 15.Es importante ponerle excitación a mi vida. & & & & & \\
\hline 16.He cometido errores en el pasado que ojalá pudieran deshacerse. & & & & & \\
\hline 17.Antes de tomar una decisión, evalúo los costos y beneficios. & & & & & \\
\hline 18. Tomar riesgos hace que mi vida no sea aburrida. & & & & & \\
\hline $\begin{array}{l}\text { 19.Me resulta difícil olvidar imágenes desagradables de mi infancia y } \\
\text { adolescencia. }\end{array}$ & & & & & \\
\hline $\begin{array}{l}\text { 20.Aun cuando estoy disfrutando el presente, tiendo a hacer } \\
\text { comparaciones con experiencias similares del pasado. }\end{array}$ & & & & & \\
\hline 21.Tomo riesgos para ponerle excitación a mi vida. & & & & & \\
\hline 22.Uno no puede planificar el futuro porque las cosas cambian mucho. & & & & & \\
\hline
\end{tabular}


23.Mi vida está controlada por fuerzas que no puedo manejar.

24.No tiene sentido preocuparse por el futuro si, de todos modos, no puedo hacer nada por ello.

25.Pienso en las cosas malas que me han ocurrido en el pasado.

26.El ser constante me permite completar mis proyectos a tiempo.

27.Me dejo llevar por la excitación del momento.

28.Me gustan las tradiciones familiares que se repiten regularmente.

29.Pienso en las cosas buenas que me he perdido en mi vida.

\section{Instrucciones para la corrección}

Para obtener las puntuaciones directas de cada orientación temporal se realiza la sumatoria de los ítems dividido la cantidad de ítems.

\section{Ítems directos: todos menos el inverso}

Completamente Falso $=1$

Bastante Falso $=2$

Neutral $=3$

Bastante Verdadero $=4$

Completamente Verdadero $=5$

Ítem inverso: 14

Completamente Falso $=5$

Bastante Falso $=4$

Neutral $=3$

Bastante Verdadero $=2$

Completamente Verdadero $=1$

PASADO NEGATIVO $=(1+9+16+19+20+25+29) / 7$

PRESENTE HEDONISTA $=(3+11+15+18+21+27) / 6$

FUTURO $=(4+6+10+13+17+26) / 6$

PASADO POSITIVO $=(2+5+8+12+14+28) / 6$

PRESENTE FATALISTA $=(7+22+23+24) / 4$ 